\title{
Training proprioception with sound: effects of real-time auditory feedback on intermodal learning
}

\author{
Shashank Ghai ${ }^{1}$, Gerd Schmitz ${ }^{1}$, Tong-Hun Hwang ${ }^{1,2}$, and Alfred O. Effenberg ${ }^{1}$ \\ ${ }^{1}$ Institute of Sports Science, Leibniz University Hannover, Germany \\ ${ }^{2}$ Institute of Microelectronic Systems, Leibniz University Hannover, Germany
}

Address for correspondence: Shashank Ghai, Institut für Sportwissenschaft, Leibniz Universität, Am Moritzwinkel 6, Hannover 30167, Germany. shashank.ghai@sportwiss.uni-hannover.de

Short title: Effects of Sonification on Intermodal Learning

\begin{abstract}
Our study analyzed the effects of real-time auditory feedback on intermodal learning during a bilateral knee repositioning task. Thirty healthy participants were randomly allocated to control $(\mathrm{n}=15)$, and experimental groups (15). Participants performed an active knee joint repositioning task for four target angles $\left(20^{\circ}, 40^{\circ}, 60^{\circ}\right.$ and $\left.80^{\circ}\right)$, bilaterally, with/without additional real-time auditory feedback. Here, the frequency of the auditory feedback was mapped to the knee's angle range $\left(0-90^{\circ}\right)$. Retention measurements were performed on the same four angles, without auditory feedback, after 15 minutes and 24 hours. A generalized knee proprioception test was performed after the 24 -h retention measurement on three untrained knee angles $\left(15^{\circ}, 35^{\circ}\right.$, and $\left.55^{\circ}\right)$. Statistical analysis revealed a significant enhancement of knee proprioception, shown as a lower knee repositioning error with auditory feedback. This enhancement of proprioception also persisted in tests performed between the 5th and 6th auditory-motor training blocks (without auditory feedback). Enhancement in proprioception also remained stable during retention measurements (15 minutes and 24 hours later). Similarly, enhancement in the generalized proprioception on untrained knee angles was evident in the experimental group. This study extends our previous findings and demonstrates beneficial effects of real-time auditory feedback to facilitate intermodal learning by enhancing knee proprioception in a persisting and generalized manner.
\end{abstract}

Keywords: perception; rehabilitation; sonification; coordination; joint position sense; motor learning 


\section{Introduction}

Acquisition of a motor skill depends on the availability of task-relevant perceptual information that can mediate motor control and performance. ${ }^{1,2}$ According to Wolpert, Diedrichsen and Flanagan ${ }^{3}$, the process of skill acquisition involves the establishment of associations between motor and sensory variables, such as internal models, which represent features of movement execution. Here, amplifying the representation of the perceptual information by the means of augmented sensory feedback, such as real-time auditory feedback can allow enhancements in performance. ${ }^{4}$ The availability of additional perceptual information might allow a performer to selectively adjust their attention towards the taskrelevant perceptual modality for effectively completing the task ${ }^{5-7}$. Moreover, such a feedback can enrich the development of perceptomotor representations by amplifying the brain's ability to integrate multiple congruent perceptual streams, thereby aiding in the formation of stable internal feed-forward models. $^{3,8}$

Research conclusively suggests that mapping a performer's action with real-time auditory feedback can enhance both the perceptuomotor representations in the brain, and motor performance. ${ }^{8-12}$ Strong influence of real-time auditory feedback on motor performance had been thought to be due to its influence over the proprioceptive modality. ${ }^{13-16}$ Hasegawa, et al. ${ }^{16}$, for instance, reported that training with auditory augmented biofeedback might facilitate the integration of auditory and proprioceptive systems. The authors suggested that the auditory system can promote a challenging, resource-dependent learning environment that might increase the reliance on proprioceptive information. Recent research by Ghai et al. ${ }^{19}$ has also demonstrated that real-time auditory feedback could influence kneeproprioceptive perceptions. The authors reported that concurrent application of auditory feedback can enhance knee joint repositioning accuracy. However, these effects were merely transient, as once the feedback was removed the proprioceptive errors returned to the levels observed before training. This goes in line with previous research reporting performance decrements with the withdrawal of augmented feedback (see guidance hypothesis ${ }^{17}$ ). According to the main reason for such performance, decrements could be over-dependency of a learner on an augmented feedback at the expense of relying on their intrinsic sources to support their performance when the feedback is removed as the retention test. ${ }^{18}$ Conventionally, a motor skill cannot be considered "learned" until retention and/or skill transfer has been demonstrated. Therefore, a lack of retainable and transferrable effects can raise serious concerns regarding the viability and robustness of an intervention.

In the research of Ghai, et al. ${ }^{19}$ two main limitations could have accounted for the lack of retainable effects. Firstly, the use of a constant or blocked training regimen. In the experiment, the participants were instructed to consecutively reposition their dominant knee 15 times, at two different target angles, each. Here, a lack of variability ( 15 continuous repetitions for $40^{\circ}$ and then $75^{\circ}$ ) could have been the main reason for performance decrements during the retention measurements. According to Cross, Schmitt and Grafton ${ }^{20}$, incorporating a variable training regimen can induce mechanisms of contextual interference, which might force a learner to effortfully reconstruct internal models in their working memory. ${ }^{21,22}$ Therefore, promoting a persistent, robust representation of the skill set in the memory systems, which could then be retained and/or transferred to another skill set. ${ }^{23,24}$

Secondly, the short training duration (5-7 min) with auditory feedback by Ghai, et al. ${ }^{19}$ could also have served as an important factor in the lack of retainable effects. ${ }^{25}$ Previous research analyzing the effects of auditory feedback on motor performance with shorter training durations such as, Dyer, Stapleton and Rodger ${ }^{26}$ have also demonstrated performance decrements during 24-h retention measurements. ${ }^{6}$ Here, the main reasons for the lack of performance retention could be interpreted from neuroimaging research by Bangert and Altenmüller ${ }^{27}$, and Ross, Barat and Fujioka ${ }^{28}$. These research outline a temporal course necessary for establishing stable intermodal auditory-sensorimotor coactivation. Bangert and Altenmüller ${ }^{27}$, for instance, analyzed cortical activation patterns during an audio-motor training session (20 minutes). The authors based on EEG measurements reported auditory-sensorimotor coactivity emerging after 20 min of training. Similarly, Ross, et al. ${ }^{28}$, reported functional neuroplastic changes (higher P2 activity and $\beta$-band oscillation) with a prolonged auditory-motor training session (30 
minutes). Several of the systematic reviews and meta-analyses have also suggested a similar temporal course for auditory-motor training regimens to allow enhancements in motor performance. ${ }^{29-34}$

In the present research, we aim to address the limitations of the experimental design used in and also elucidate the influence of auditory feedback in motor learning. An expanded intermodal auditoryproprioceptive training protocol has been developed to investigate the efficacy of real-time auditory information on proprioceptive motor learning. First and foremost, we extend the length of training duration with auditory feedback by incorporating more target angles (four vs two), a higher number of auditory-motor knee repositioning trials ( 288 vs 30 ) and with bilateral distribution. Second, we induce variability in the training protocol by inducing randomized performance on four target angles, as compared to a consecutive performance by Ghai, et al. ${ }^{19}$. We also aim to deduce a temporal course for the development of auditory-motor coupling by incorporating pure proprioceptive measurements (without auditory feedback) between audio-motor training blocks. Finally, we also test the robustness of the intervention by analyzing delayed retention on trained angles and generalized proprioceptive performance on untrained angles after the completion of the experiment.

In the present study, we propose two main hypotheses (1) based on extended auditory-motor training duration we expect a persistent enhancement of knee-proprioceptive accuracy (enhanced kneeproprioceptive performance) be maintained on the trained angles in the absence of auditory feedback (immediately after $15 \mathrm{~min}$ and $24 \mathrm{~h}$ ) and (2) we expect that the enhancements of knee-proprioception accuracy will be demonstrated on untrained repositioning angles of the same knee. This work for the first time examines these two aspects of real-time auditory feedback on intermodal learning.

\section{Methods}

\section{Experimental design}

Participants were randomly placed in equal numbers to the control $(\mathrm{n}=15)$ and the experimental (15) groups. In each group, participants carried out active knee-joint repositioning tasks, bilaterally for four different angles $20^{\circ}, 40^{\circ}, 60^{\circ}$ and $80^{\circ}$. The experimental group received movement induced real-time auditory feedback whereas the control group received ocean wave noise to control for possible effects of an unspecific acoustic stimulus. The design (Fig. 1) consisted of nine treatment blocks, which were preceded and followed by passive knee proprioceptive tests (PPTs). Repositioning tasks without any auditory feedback were performed on 1 st, 3rd, 5th, and 7 th blocks. These blocks analyzed proprioceptive performance on the four target angles of $20^{\circ}, 40^{\circ}, 60^{\circ}$, and $80^{\circ}$. Thereafter, the 8 th and the 9th block analyzed proprioceptive performance on the same four angles in delayed retention measurements after $15 \mathrm{~min}$ and $24 \mathrm{~h}$ of the final test. Auditory feedback was provided in the $2 \mathrm{nd}$, 4th, and 6th blocks. After the final retention measurement at the 9th block (post $24 \mathrm{~h}$ ) generalized proprioceptive accuracy was analyzed on three untrained angles of $15^{\circ}, 35^{\circ}$, and $55^{\circ}$.

\section{Participants}

Thirty participants, recruited from the department of sports science, were randomly allocated to the control ( 7 males and 8 females; age (mean $\pm \mathrm{SD}$ ): $25.3 \pm 3.2$ years), and the experimental group (6 males and 9 females; $23.2 \pm 3.0$ years) volunteered to participate in the study. All participants were selfreported as healthy with no history of significant hip, knee, or back injuries. Ethical approval was obtained from the Ethics Committee of the Leibniz University Hannover, and participants gave a written informed consent for participating in the study. All participants underwent a baseline auditory test (HTTS Audiometry) to check for normal hearing ability. All participants were paid $€ 16$ for their participation.

\section{Procedure}

Participants were comfortably seated with their feet in the air, their back resting against a wall, and their pelvis stabilized. ${ }^{19,35}$ During the sitting position, the knee joint was maintained at the right angle 
(Supplementary Figure 1, online only). This position of the knee joint was considered as $0^{\circ}$ and further extension from this position onwards was referred to as a positive change in the angular values. Participants wore wireless headphones (Sennheiser ${ }^{\circledR}$, Wedemark, Germany), and were blindfolded to eliminate visual information. Initially, a familiarization session was performed to accustom the participants with the four target angles $\left(20^{\circ}, 40^{\circ}, 60^{\circ}\right.$, and $\left.80^{\circ}\right)$ they had to perform during the experiment. Here, the experimenter passively moved the dominant leg to previously identified target angles in an open kinetic chain and held at each angle for two seconds to allow the participant to memorize the position. ${ }^{36}$ This process was repeated again on the non-dominant leg. The experimenter asked the participants to memorize each target position as angle 1: $20^{\circ}$, angle $2: 40^{\circ}$, angle $3: 60^{\circ}$, and angle 4: $80^{\circ}$, on both legs. Participants received no information concerning the actual values of the angles they were performing.

After the familiarization session, a passive knee repositioning test was performed for all the four angles $\left(20^{\circ}, 40^{\circ}, 60^{\circ}\right.$, and $\left.80^{\circ}\right)$, bilaterally. Here, the experimenter passively positioned the leg at one of the four angles and held for five seconds. Thereafter, the experimenter returned the leg at the initial $0^{\circ}$ position. Thereafter, the participants were instructed to actively reposition their leg at the specific angle. This was repeated for all the four target angles, bilaterally (see pre-test, Fig. 1).

Further, in the 1st block of the experimental setup (see proprioceptive test (PPT) 1, Fig. 1) participants were verbally instructed by the experimenter to perform the same four target angles (angle 1:20 ${ }^{\circ}$, angle 2: $40^{\circ}$, angle $3: 60^{\circ}$, and angle $4: 80^{\circ}$ ), with no auditory feedback, and without any prior passive knee repositioning instruction. The verbal instructions for the performance of angles were randomized as right leg angle 1, right leg angle 4, right leg angle 3, and so on. A total of 32 repetitions were performed by the right leg. This process was again repeated by the left leg. A total of 64 repetitions were performed in this block, which took about 8-10 minutes. Furthermore, before the commencement of the 2 nd block, participants were introduced to the auditory feedback (the control group was introduced to an ocean wave noise). Here, the experimenter first passively repositioned the legs at the four angles, bilaterally with the auditory feedback. This was performed to ensure that the participants could associate the target angles (angle 1: $20^{\circ}$, angle 2: 40 , angle 3: $60^{\circ}$, and angle 4: $80^{\circ}$ ) with their respective sounds (Supplementary File 2, online only). After that, the participants were verbally instructed to reposition their knee joints by themselves, in the presence of auditory feedback (see R-AF 1, Fig. 1). Here as well, the verbal instructions for the performance of angles were randomized as right leg angle 4, right leg angle 3, and right leg angle 1, and so on. This process was again repeated on the left leg. A total of 96 repetitions were performed in this block ( 48 right +48 left). The duration of the training blocks (R-AFs) lasted for 15-20 minutes. Here, both the experimental group and the control group trained with an identical duration.

After this, the 3rd block analyzed proprioceptive accuracy without any auditory feedback (see PPT 2, Fig. 1). Like the 1 st block the participants were verbally instructed by the experimenter to actively reposition their knee joints at the four target angles $\left(20^{\circ}, 40^{\circ}, 60^{\circ}\right.$ and $\left.80^{\circ}\right)$ in a randomized order. The procedure, total number of repetitions, and duration were identical to the 1 st block. The 4th block was an auditory-motor training block (see R-AF 2, Fig. 1). Here, auditory feedback was present. Like the 2nd block, the experimenter initially repositioned the participant's knee passively with the auditory feedback. Thereafter, the participants were verbally instructed, in a randomized order to reposition their knee joints. The procedure, total number of repetitions, and duration were identical to the 2nd block.

The 5th block analyzed proprioceptive accuracy without any auditory feedback (see PPT 3, Fig. 1). Like the 1st and 3rd blocks, the participants were verbally instructed, in a randomized order, to actively reposition their knee joints at the four angles $\left(20^{\circ}, 40^{\circ}, 60^{\circ}\right.$ and $\left.80^{\circ}\right)$. The procedure, total number of repetitions, and duration were identical to the 1 st and 3rd blocks. Thereafter, the 6 th block was a training block (see R-AF 3, Fig. 1). Here, auditory feedback was present. Like the 2nd and 4th blocks, the experimenter initially repositioned the participant's knee passively with the auditory feedback. Thereafter, the participants were verbally instructed, in a randomized order to actively reposition their knee joints. The procedure, total number of repetitions, and duration were identical to the 2 nd and 4 th blocks. The 7th block analyzed the proprioceptive accuracy in a final step without any auditory 
feedback (see PPT Final, Fig. 1). Like the 1st, 3rd and 5th blocks, the participants were verbally instructed, in a randomized order to actively reposition their knee joints at the four target angles $\left(20^{\circ}\right.$, $40^{\circ}, 60^{\circ}$ and $80^{\circ}$ ). The procedure, total number of repetitions, and duration were identical to the $1 \mathrm{st}$, $3 \mathrm{rd}$, and 5th blocks.

Thereafter, the 8th block analyzed the retention of performance after 15 min of completion of the 7 th block (PPT final), without any auditory feedback (see RET 15min, Fig. 1). Like the 1st, 3rd 5th and 7th block, the participants were verbally instructed, in a randomized order to actively reposition their knee joints at the four target angles $\left(20^{\circ}, 40^{\circ}, 60^{\circ}\right.$, and $\left.80^{\circ}\right)$. The procedure, total number of repetitions, and duration were identical to the $1 \mathrm{st}, 3 \mathrm{rd}$, 5th, and 7th blocks. The 9th block analyzed the retention of performance after $24 \mathrm{~h}$ of completion of the 7 th block, without any auditory feedback (see RET $24 \mathrm{~h}$, Fig. 1). Like the $1 \mathrm{st}, 3 \mathrm{rd}, 5 \mathrm{th}, 7^{\text {th }}$, and 8 th blocks, the participants were verbally instructed, in a randomized order to actively reposition their knee joints at the four target angles $\left(20^{\circ}, 40^{\circ}, 60^{\circ}\right.$ and $80^{\circ}$ ). The procedure, total number of repetitions, and duration were identical to the $1 \mathrm{st}, 3 \mathrm{rd}, 5 \mathrm{th}, 7 \mathrm{th}$ and 8th block.

Finally, after the completion of the 24-h retention measurement, transferability of skill was analyzed in a generalized PPT. Here, the participants' performance on three completely untrained angles $\left(15^{\circ}, 35^{\circ}\right.$, and $55^{\circ}$ ) was tested (see G-test, Fig. 1). Like the pre-test, the experimenter first passively repositioned the knee at one of the target angles and held the position for five seconds. Thereafter, the participants were instructed to actively reposition their leg at the specific angle. This process was repeated for all the three target angles $\left(15^{\circ}, 35^{\circ}\right.$, and $\left.55^{\circ}\right)$ bilaterally. Figure 1 illustrates the entire experimental procedure. Moreover, a detailed breakdown of the blocks in terms of total number of repetitions performed, the presence of auditory feedback and target angles performed has been illustrated in Supplementary File 3, online only). The experimental protocol lasted approximately for 100-120 minutes.

The auditory feedback used in this experiment was identical to that used by Ghai, et al. ${ }^{19}$. The changes in angles from $0^{\circ}$ to $90^{\circ}$ of full knee-extension was mapped to a frequency spectrum ranging from 120 $\mathrm{Hz}$ to $300 \mathrm{~Hz}$. A sample of auditory feedback has been provided as Supplementary File 2 (online only). The mapping functions as a mathematical equation have been mentioned by Ghai, et al. ${ }^{19}$.

\section{Kinematic analysis}

XSENS MVN Biomech (XSENS Technologies B.V, Netherlands) in a lower body configuration mode was used to assess knee joint angles. Seven wireless inertial measurement units were positioned by the experimenter on the participants using Velcro straps. The inertial measurement units were positioned on the sacrum, the lateral side of the femoral shaft, the medial surface of the tibia, and the talus. With the wireless data transmission, kinematic motion was recorded in a three-dimensional Cartesian coordinate system at a $60-\mathrm{Hz}$ sampling frequency. The knee joint angle data are analyzed by a Xsens ${ }^{\circledR}$ MVN Studio version 4.3 software (Xsens, the Netherlands) that recorded the movement and the kinematic data in MVN file format. Thereafter, the repositioning data for each trial were matched with the MVN data recordings and were extracted manually by two researchers. The absolute error was calculated to quantify the magnitude of the repositioning error. ${ }^{35}$ Studies have reported high reliability and validity of Xsens motion capture system for joint angular data measurement. ${ }^{37,38}$ The total number of trials performed in this experiment were 742 (Supplementary File 3, online only). No trial was excluded from the final analysis.

\section{Statistical analysis}

Statistical analyses were performed using Statistica (V. 12. StatSoft, Hamburg, Germany). According to research question 1, we wanted to investigate the changes of proprioceptive accuracy over time induced by auditory feedback training as well as whether changes persist in the retention tests after 15 minutes and 24 hours. Therefore, we submitted repositioning errors (the dependent measure) to a two- 
way ANOVA with the between-subject factor group (Experimental/Control) and the within-subject factor block (PPT 1, R-AF 1, PPT 2, R-AF 2, PPT 3, R-AF 3, PPT final, RET 15 min, and RET 24 hours). A post-hoc Bonferroni-test allowed us to perform pairwise group comparisons for each block to scrutinize whether group differences emerge over time. Furthermore, it became possible to perform within-group comparisons between all proprioceptive blocks without auditory feedback (PPT 1, PPT 2, PPT 3, PPT Final, RET $15 \mathrm{~min}$, and RET $24 \mathrm{~h}$ ) to test whether retention measures (RET $15 \mathrm{~min}$, RET $24 \mathrm{~h}$ ) differ from PPT1 and PPT Final. Research question two was analyzed by a two-way ANOVA with the between-subject factor group and the within-subject factor test (pre-test and G-Test). Effect sizes of the independent variables were expressed using partial eta squared $\left(\eta_{\mathrm{p}}{ }^{2}\right)$, with effect sizes $<$ 0.01 considered to be small, effect sizes of 0.06 considered to be medium and effect sizes $>0.14$ considered to be large. The Bonferroni correction was performed for post-hoc analyses. The overall significance level was set to $5 \%$.

\section{Results}

\section{Effect of audio-motor training on proprioceptive accuracy}

Knee repositioning errors of both groups are shown in Figure 2 (for descriptive statistics see Supplementary Files 4 and 5, online only). Both groups started at the same level but diverged from the second block on (R-AF 1). This was due to the performance increase of the experimental group, which became evident when participants were provided with auditory feedback for the first time (R-AF 1). Accordingly, an ANOVA yielded significance for the main effects as well as their interaction (group: $\mathrm{F}(1,28)=84.02, p<0.001, \eta_{p}{ }^{2}=0.75$; block: $F(8,224)=3.24, p<0.001, \eta_{\mathrm{p}}{ }^{2}=0.17$; block $\mathrm{x}$ group: $\left.F(8,224)=7.75, p<0.001, \eta_{p}{ }^{2}=0.22\right)$. Bonferroni-adjusted post-hoc comparisons revealed significantly better performance in blocks R-AF1, R-AF2 and R-AF3 for those participants who were provided with the auditory feedback and not the control stimulus (all $p<0.001$ ).

With respect to proprioceptive accuracy, groups did not differ significantly at the first two PPTs (PPT 1: $p>0.999$; PPT 2: $p>0.915$ ), but at all other PPTs (PPT 3, PPT Final, RET 15 min, RET 24 h). Furthermore, participants in the experimental group maintained their proprioceptive accuracy from PPT 3 on. In more detail, PPT3, PPT final, RET $15 \mathrm{~min}$, and RET $24 \mathrm{~h}$ did not differ significantly from each other, but they all differed significantly from PPT 1 (all $p<0.001$ ) and PPT 2 (all p's at least $p<0.05$ ). In the control group, no differences were significant (all $p>0.05$ ).

\section{Generalization effect}

Repositioning errors of the pre-test and the generalization test (G-test) are illustrated in Figure 3. Prior to feedback exposure, both groups had the same level with respect to repositioning accuracy, which diverged post exposure. Accordingly, an ANOVA confirmed a significant group effect $(F(1,28)=17.33$, $\left.p<0.001, \eta_{p}{ }^{2}=0.38\right)$ as well as a significant group $\mathrm{x}$ test interaction $\left(F(1,28)=24.42, p<0.001, \eta_{p}{ }^{2}=\right.$ 0.47). A post-hoc test to this interaction showed that between-group differences were not significant in the pre-test $(p>0.999)$, but in the generalization test $(p<0.001)$. Furthermore, generalized enhancement in knee proprioception was significant in the experimental $(p=0.002)$, but not in the control group $(p$ $=0.051)$.

\section{Discussion}

This experiment for the first time analyzed the effects of real-time auditory feedback on kneeproprioceptive learning. Here, active knee repositioning trials were performed bilaterally for four target angles $\left(20^{\circ}, 40^{\circ}, 60^{\circ}\right.$, and $\left.80^{\circ}\right)$, bilaterally, with/without additional real-time auditory feedback. The main findings of our study are:

a) Real-time auditory feedback significantly enhanced knee proprioception (lower repositioning errors). 
b) Significant enhancements in knee proprioception were observed in the experimental group after 3040 min of training, evident from the PPT 3 and were also evident in the final PPT.

c) Significant enhancements in knee proprioception accuracy were also evident in the experimental group during delayed retention measurements at post 15 minutes and 24 hours.

d) Significant enhancements in knee proprioception were also demonstrated in the experimental group during a generalized knee PPT on completely untrained angles $\left(15^{\circ}, 35^{\circ}\right.$, and $\left.55^{\circ}\right)$.

In agreement with our previous study beneficial effects of real-time auditory feedback on proprioception were observed in the training blocks (R-AF, Figs. 1 and 2). ${ }^{19}$ The mechanisms underlying such benefit are likely to be multifactorial. For instance, the auditory feedback could have provided external guidance for repositioning, ${ }^{12}$ enhanced error feedback, ${ }^{6}$ enhanced multisensory integration, ${ }^{39}$ strengthened perceptuomotor representations, ${ }^{40}$ allowed selective attentional allocation, ${ }^{41,42}$ and more 43, 44 (for a detailed discussion see Ref. ${ }^{19}$ ).

In this study, our focus was to address the limitations of Ghai, et al. ${ }^{19}$, by demonstrating kneeproprioceptive enhancements during retention and generalized PPTs. We analyzed whether modifications in terms of variability in training, and prolonged training duration could influence kneeproprioceptive learning. Firstly, we adapted our auditory-motor training intervention in terms of duration by increasing the number of angles (four), and the number of auditory-motor training repetitions (288), performed bilaterally. In agreement with our hypothesis, enhancement in kneeproprioceptive accuracy were observed with the prolongation of auditory-motor training. We report significant enhancements in proprioception accuracy observed from the PPT 3 (Fig. 1). These enhancements in proprioception accuracy were evitable after two blocks of auditory-motor training (RAF1, R-AF2), which lasted for approximately 30-40 minutes (Fig. 2). This conclusion is drawn on the basis that a single R-AF1 auditory-motor training block (15-20 min) allowed only transient enhancements in knee-proprioception accuracy (similar to our previous study ${ }^{19}$ ). Nevertheless, after the second blocks of auditory-motor training (R-AF1 and R-AF2) the enhancements in proprioception were stable and were also evident in the final proprioceptive and retention tests (PPT Final, RET 15 min, RET 24 hours). However, this was not the case for the control group, which received task-irrelevant ocean wave noise. Here, the proprioceptive performance remained largely unchanged during the entire course of training. Inference for this different time-dependent development of proprioceptive accuracy between the experimental and the control groups could be affirmed to the findings of Auksztulewicz, Friston and Nobre ${ }^{45}$. The authors reported that task-relevant sensory information could allow modulation in behavior in terms of enhanced spatial-temporal predictability and discrimination. On the contrary, task-irrelevant feedback adversely affected this predictive mechanism, possibly because of the wasteful processing by cognitive resources. ${ }^{45}$ Therefore, explaining the differential time-dependent changes in proprioceptive perceptions between the experimental and the control groups.

Likewise, the findings concerning time-dependent enhancement in proprioceptive accuracy in the experimental group are also in line with the results of neuroimaging studies outlining a temporal course for the establishment of auditory-sensorimotor coactivation. ${ }^{27,}{ }^{28}$ Furthermore, with respect to our retention measurements ( $15 \mathrm{~min}$ and $24 \mathrm{~h}$ later), findings of Tremblay, et al. ${ }^{46}$ are referred to. Tremblay, et al. ${ }^{46}$ suggested that repeated exposure of an auditory stimulus during audio-motor training might effectively prime the auditory system, thereby allowing retention of skill even after a long period of time. ${ }^{46}$ Similarly, Hasegawa, et al. ${ }^{16}$, for instance, in their study revealed that training with auditory biofeedback led to robust, retainable enhancements in spatial and temporal components of postural stability.

Additionally, in the present experiment, we demonstrate the robustness of auditory-motor coupling in a generalized PPT. Here, participants in the experimental group demonstrated a "generalized" enhancement in knee proprioception on completely untrained angles after $24 \mathrm{~h}$ of the experiment. Here, relevant to the findings of Bangert and Altenmüller ${ }^{27}$, we presume that auditory-motor training could have facilitated the development of an interfaced mapping (intermodal coupling between the auditory and proprioceptive systems). In simpler terms, as the participants performed knee extensions from the 
initial starting position of $0^{\circ}$ to the four target angles $\left(20^{\circ}, 40^{\circ}, 60^{\circ}\right.$, and $\left.80^{\circ}\right)$. We believe the participants could have developed an implicit, interfaced audio-proprioceptive map for the entire performed a range of motion from $0^{\circ}$ to $80^{\circ}$. This could also mean that the participants not only learned to reproduce the pitch precisely but learned a more precise use of proprioceptive information from the knee joint. This eventually could have allowed enhanced performance on both the trained and untrained angles.

Furthermore, modifications in terms of variability (randomized performance of target angles, and a leg) were also introduced to our previous training paradigm. ${ }^{19}$ This inclusion of variability could have also played an important role in maintaining proprioceptive performance during retention and generalized PPTs. ${ }^{24,47,48}$ Several reasons can be asserted for this enhancement in motor performance based on the theory of contextual interference. According to Battig ${ }^{22}$, a variable training paradigm could have allowed a learner to encode different strategies such as using multiple routes to acquire a new skill. This could then have promoted a more elaborate memory representation as compared to single elaborate strategies such as constant training. ${ }^{22}$ Furthermore, this strategy could allow an enhanced retention and skill transfer by promoting retrieval of a learned skill set through multiple retrieval routes established during variable training. Moreover, a variable training regimen might also promote effortful execution on the behalf of learner, eventually developing a stronger representation of performed motor skill set. This, then might promote development of efficient action plan reconstruction which can allow enhancements in performance during both retention and motor skill transfer tests. ${ }^{49}$ Neuroimaging studies also confirm that the indulgence of variability during training can promote a broader network of sensorimotor, premotor-parietal networks, and subcortical areas as compared to constant training. ${ }^{20,50}$ Likewise, longitudinal analysis demonstrated stable or increased activation in areas associated with motor preparation, sequencing, and response selection in the group training variably. ${ }^{20}$ In our previous study, we assumed that a constant training on the two target angles $\left(40^{\circ}\right.$ and $\left.75^{\circ}\right)$ could have been one of the main reason for the lack of retainable effects in the consecutive retention block. Nevertheless, in the present study retainable, and generalized enhancements in the proprioceptive performance might also have been due to the indulgence of variability in auditory-motor training regimen.

As an additional and important aspect, we postulate that an intermodal integration of auditory and proprioceptive information could have further enhanced the spatial contingency, ${ }^{51}$ as was demonstrated in the current repositioning task. According to Effenberg, et al. ${ }^{8}$, convergent sensory feedback, which share a high level of spatiotemporal proximity can get implicitly fused in order to promote intermodal learning (in this case auditory and proprioceptive). ${ }^{10}$ Here, additional inference can be drawn from literature emphasizing on the importance of intermodal knowledge for obtaining spatial knowledge of body in space. ${ }^{52,53}$ Likewise, evidence from neuroimaging studies also supports the notion that a high level of stimulus-response consistency (meaningful organization of perceptual and motion events) can promote sensorimotor coactivations, ${ }^{27}$ and motor priming. ${ }^{54}$ Therefore, we propose that in the current study, the convergence of the perceptual modalities (auditory-proprioceptive) due to the comprehensive audio-motor execution could have allowed a feature overlap between perception and action, ${ }^{10,} 55,56$ and/or supported the development of important amodal relations. ${ }^{53}$ This then could have provided a platform for the development of consistent sensorimotor representations perceived in a unified manner, therefore enhancing intermodal learning. ${ }^{51,53}$ In terms of neuroplastic changes that might have taken place with our auditory-motor training paradigm, we interpret our results from the findings of Classen, et al. ${ }^{57}$. Based on the findings of these authors we presume that the mechanisms of short-term potentiation were involved in our present study. ${ }^{57} \mathrm{~A}$ major limitation persisted in our study in terms of the generalization proprioception test. Here, we compared initial performance of four angles $\left(20^{\circ}, 40^{\circ}\right.$, $60^{\circ}$, and $80^{\circ}$ ) with three untrained angles (G-test, $\left.15^{\circ}, 35^{\circ}, 55^{\circ}\right)$. This indirect comparison might limit our interpretations as to the generalized proprioceptive influence of auditory-motor training on terminal knee angles of $>55^{\circ}$.

In conclusion, we report significant enhancement of knee proprioception accuracy with real-time auditory feedback. Moreover, we report that modification of an auditory-motor training paradigm, in terms of longer training duration, and variable training regimen can allow retainable (post 15-min, post24 hour) and generalized" (skill transfer on untrained angles) enhancements in proprioceptive accuracy. 
In terms of practical applications, we strongly refer to research outlining the beneficial aspects of joint position sense (similar to the present joint repositioning task) in musculoskeletal disorders. ${ }^{58,59}$ Research suggests that the sense of joint position possibly mediates thixotropic changes in muscle spindles, and slow-adapting mechanoreceptors. ${ }^{60-62}$ Evidence from knee studies also confirm the predominant role of mechanoreceptors situated in the ligamentous structures of the knee joint (especially cruciate ligaments). ${ }^{61,62}$ Therefore, enhancements observed in the perception of knee joint position sense in the current study could be applicable both as a prophylaxis, ${ }^{58,}{ }^{63}$ as well as a rehabilitation strategy for many knee disorders such as, meniscal tear, cruciate ligament injuries, knee arthroplasty, and patellofemoral pain syndrome. ${ }^{58,64-67}$

Finally, a plausible explanation for our findings can be the auditory system's high-resolution capability of pitch differences and temporal features. Higher auditory resolution could have trained the comparably lower resolution proprioceptive system in both domains via intermodal referencing. Such enhancements that are based on intermodal processing between modalities of different perceptual characteristics could be addressed, in this context as core mechanisms of intermodal learning. Here, the feedback can simultaneously assist in shaping the perceptuomotor representations - without the need of attention and higher cognitive resources. ${ }^{8,68}$

\section{Acknowledgement}

The co-author would like to thank student assistant Mr. Pascal Moszczynski for his assistance during the experimental procedure. Parts of this work was supported by the European Commission grant H2020-FETPROACT-2014 No. 641321.

\section{Competing interests}

The authors declare no competing interests.

\section{Author contributions}

A.O.E., S.G. together with G.S. developed the research question. S.G., A.O.E and G.S. developed the research paradigm. S.G. conducted the experiment, collected the data and wrote main parts of the paper. G.S. performed the statistical analysis supported by A.O.E. and S.G. and contributed to the results section. T.H. was responsible for technical implementing and customization of the sonification system. A.O.E. supervised the project. All authors critically revised the paper. 


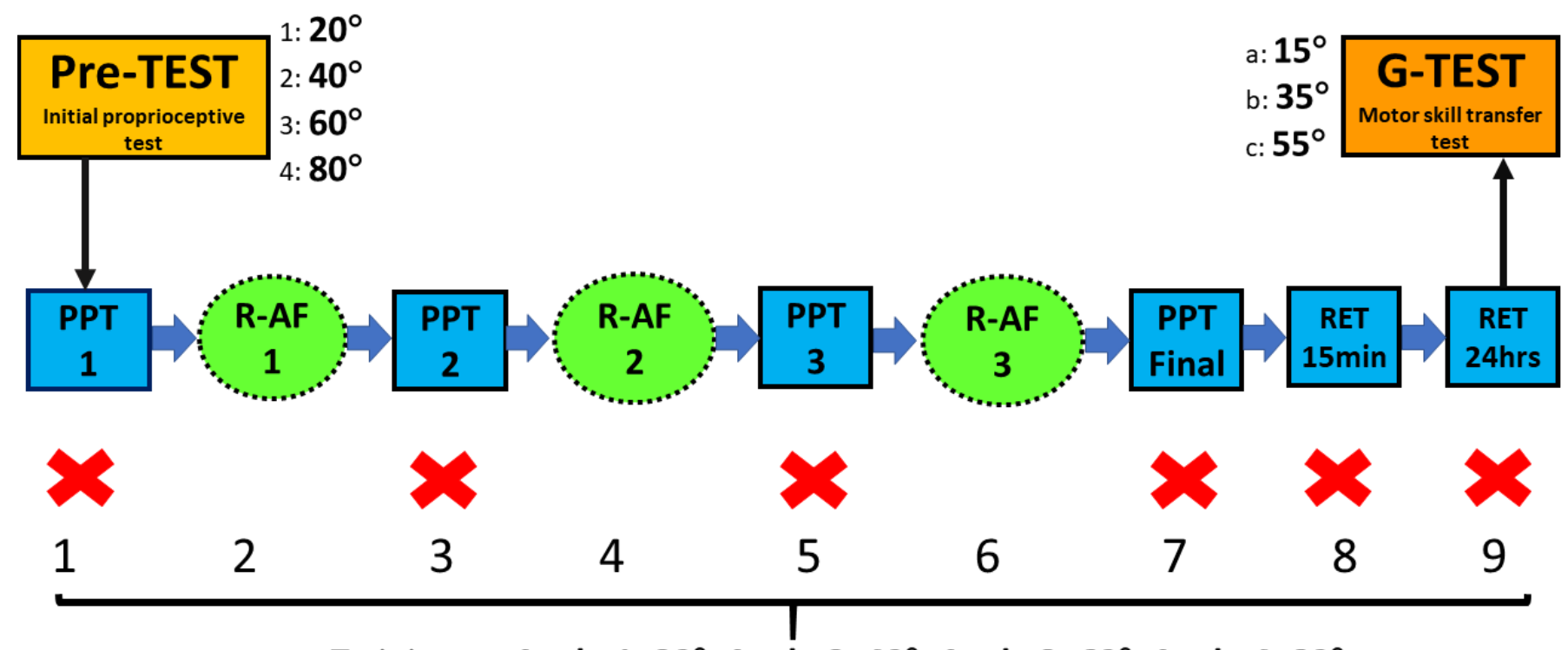

Training on Angle 1: $20^{\circ}$, Angle 2: $40^{\circ}$, Angle 3: $60^{\circ}$, Angle 4: $80^{\circ}$

$\square \& \square \rightarrow$ No sound $\quad \because \rightarrow$ Sound present

Figure 1. Experimental design. Green blocks represent training phase with real-time auditory feedback (RAF1, R-AF2, R-AF3), blue blocks represent repositioning blocks without auditory feedback (PPT-1, PPT2, PPT-3, PPT-Final) and subsequent retention test blocks (RET $15 \mathrm{~min}$, RET $24 \mathrm{hrs)} \mathrm{without} \mathrm{auditory}$ feedback. The control group received ocean wave noise during the Green training blocks. (Pre-test: Initial PPT, PPT: verbal repositioning test without auditory feedback, RET 15 min: a15-min retention, RET 24 h: a-24-hour retention test, G-test: generalized PPT)

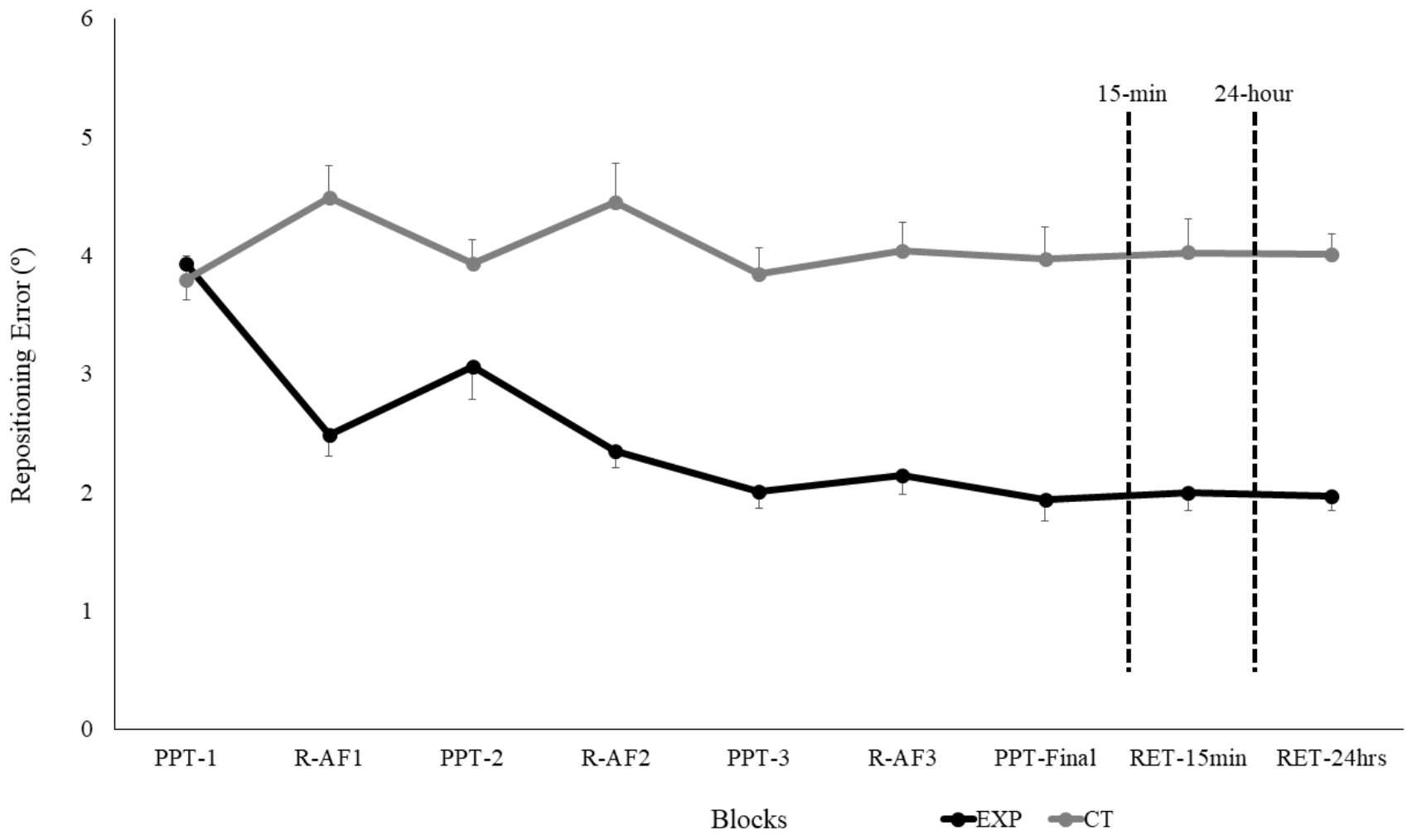


Figure 2. Absolute mean and standard error of repositioning error $\left(^{\circ}\right)$ for the mean of four angles in control, experimental group (Darkened black line represents experimental group. Darkened grey line represents control group, PPT: verbal repositioning test, R-AF: training block with a real-time auditory feedback, RET 15 min: a 15-min retention, RET 24 h: a 24-hour retention test).

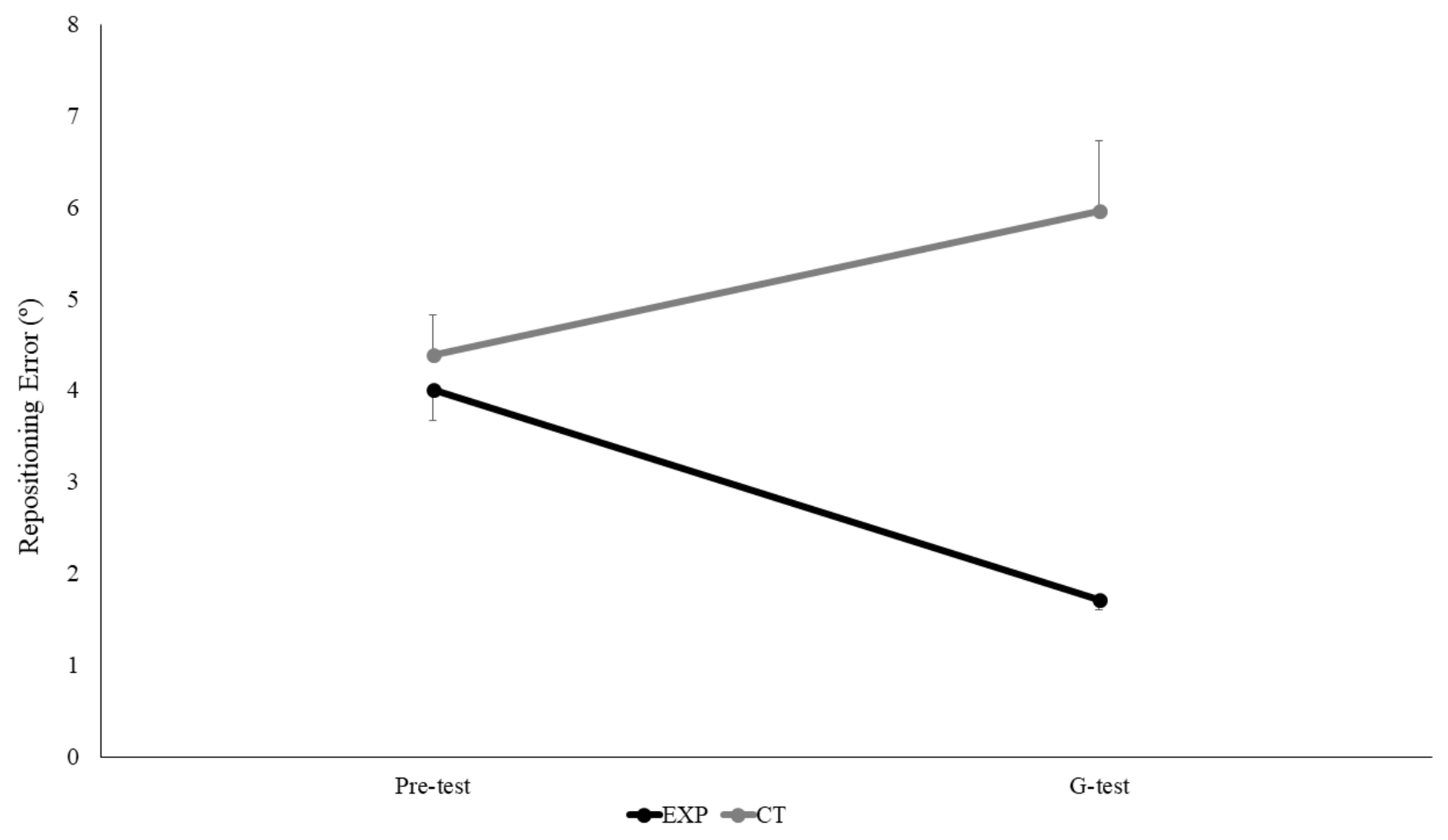

Figure 3. Absolute mean and standard error of repositioning error $\left(^{\circ}\right)$ for the mean of four angles $\left(20^{\circ}, 40^{\circ}\right.$, $60^{\circ}$ and $\left.80^{\circ}\right)$ in pre-test condition, and three untrained $\left(15^{\circ}, 35^{\circ}\right.$, and $\left.55^{\circ}\right)$ angles in generalized-test condition for both experimental and control groups. 


\section{References}

1. Mechsner, F., D. Kerzel, G. Knoblich, et al. 2001. Perceptual basis of bimanual coordination. Nature. 414: 69.

2. Newell, K.M. 1991. Motor skill acquisition. Annual review of psychology. 42: 213-237.

3. Wolpert, D.M., J. Diedrichsen \& J.R. Flanagan. 2011. Principles of sensorimotor learning. Nature. Rev. Neurosci. 12: 739-751.

4. Effenberg, A.O. 2004. "Using sonification to enhance perception and reproduction accuracy of human movement patterns". In Internation Workshop on Interactive Sonification, Vol. 2004: 1-5.

5. Gibson, E.J. 1969. Principles of perceptual learning and development. Appleton Century Crofts. New York.

6. Sigrist, R., G. Rauter, R. Riener, et al. 2013. Augmented visual, auditory, haptic, and multimodal feedback in motor learning: a review. Psychon. Bull. Rev. 20: 21-53.

7. Ronsse, R., V. Puttemans, J.P. Coxon, et al. 2011. Motor Learning with Augmented Feedback: Modality-Dependent Behavioral and Neural Consequences. Cereb. Cortex. 21: 1283-1294.

8. Effenberg, A.O., U. Fehse, G. Schmitz, et al. 2016. Movement sonification: Effects on motor learning beyond rhythmic adjustments. Front. Neurosci. 10: 219.

9. Sigrist, R., G. Rauter, L. Marchal-Crespo, et al. 2015. Sonification and haptic feedback in addition to visual feedback enhances complex motor task learning. Exp. Brain. Res. 233: 909-925.

10. Effenberg, A.O. \& G. Schmitz. 2018. Acceleration and deceleration at constant speed: systematic modulation of motion perception by kinematic sonification. Ann. N. Y. Acad. Sci. 1425: 56-59.

11. Danna, J., M. Fontaine, V. Paz-Villagrán, et al. 2015. The effect of real-time auditory feedback on learning new characters. Hum. Mov. Sci. 43: 216-228.

12. Dyer, J.F., P. Stapleton \& M.W.M. Rodger. 2017. Advantages of melodic over rhythmic movement sonification in bimanual motor skill learning. Exp. Brain. Res. 235: 3129-3140.

13. Danna, J. \& J.-L. Velay. 2017. On the Auditory-Proprioception Substitution Hypothesis: Movement Sonification in Two Deafferented Subjects Learning to Write New Characters. Front. Neurosci. 11: 137.

14. Scholz, D.S., S. Rhode, M. Großbach, et al. 2015. Moving with music for stroke rehabilitation: a sonification feasibility study. Ann. N. Y. Acad. Sci. 1337: 69-76.

15. Altenmüller, E., J. Marco-Pallares, T. Münte, et al. 2009. Neural Reorganization Underlies Improvement in Stroke-induced Motor Dysfunction by Music-supported Therapy. Ann. N. Y. Acad. Sci. 1169: 395-405.

16. Hasegawa, N., K. Takeda, M. Sakuma, et al. 2017. Learning effects of dynamic postural control by auditory biofeedback versus visual biofeedback training. Gait. Posture. 58: 188-193.

17. Schmidt, R.A. 1991. "Frequent augmented feedback can degrade learning: Evidence and interpretations". In Tutorials in motor neuroscience: 59-75. Springer.

18. Magill, R.A. \& D.I. Anderson. 2007. Motor learning and control: Concepts and applications. McGraw-Hill New York.

19. Ghai, S., G. Schmitz, T.-H. Hwang, et al. 2018. Auditory Proprioceptive Integration: Effects of RealTime Kinematic Auditory Feedback on Knee Proprioception. Front. Neurosci. 12.

20. Cross, E.S., P.J. Schmitt \& S.T. Grafton. 2007. Neural substrates of contextual interference during motor learning support a model of active preparation. J. Cogn. Neurosci. 19: 1854-1871.

21. A. Immink, M. \& D.L. Wright. 1998. Contextual interference: A response planning account. Quat. J. Exp. Psychol. A. 51: 735-754.

22. Battig, W.F. 1979. The flexibility of human memory. Levels of processing and human memory, Lawrence Erlbaum Associates, Hillsdale, NJ. 23-44.

23. Li, Y. \& D.L. Wright. 2000. An Assessment of the Attention Demands during Random- and Blocked-Practice Schedules. Quat. J. Exp. Psychol. A. 53: 591-606.

24. Müssgens, D.M. \& F. Ullén. 2015. Transfer in motor sequence learning: effects of practice schedule and sequence context. Front. Hum. Neurosci. 9: 642.

25. Sigrist, R., J. Schellenberg, G. Rauter, et al. 2011. Visual and auditory augmented concurrent feedback in a complex motor task. Presence: Teleoperators. Virtual. Environ. 20: 15-32. 
26. Dyer, J., P. Stapleton \& M. Rodger. 2017. Transposing musical skill: sonification of movement as concurrent augmented feedback enhances learning in a bimanual task. Psychol. Res. 81: 850-862.

27. Bangert, M. \& E.O. Altenmüller. 2003. Mapping perception to action in piano practice: a longitudinal DC-EEG study. BMC. Neurosci. 4: 26.

28. Ross, B., M. Barat \& T. Fujioka. 2017. Sound-Making Actions Lead to Immediate Plastic Changes of Neuromagnetic Evoked Responses and Induced beta-Band Oscillations during Perception. J. Neurosci. 37: 5948-5959.

29. Nascimento, L.R., C.Q. de Oliveira, L. Ada, et al. 2015. Walking training with cueing of cadence improves walking speed and stride length after stroke more than walking training alone: a systematic review. J. Physiother. 61: 10-15.

30. Ghai, S., I. Ghai, G. Schmitz, et al. 2018. Effect of rhythmic auditory cueing on parkinsonian gait: A systematic review and meta-analysis. Sci. Rep. 8: 506.

31. Ghai, S., I. Ghai \& A.O. Effenberg. 2017. Effect of rhythmic auditory cueing on aging gait: a systematic review and meta-analysis. Aging. Dis. 131-200.

32. Ghai, S. 2018. Effects of Real-Time (Sonification) and Rhythmic Auditory Stimuli on Recovering Arm Function Post Stroke: A Systematic Review and Meta-Analysis. Front. Neurol. 9.

33. Ghai, S. \& I. Ghai. 2018. Effects of Rhythmic Auditory Cueing in Gait Rehabilitation for Multiple Sclerosis: A Mini Systematic Review and Meta-Analysis. Front. Neurol. 9.

34. Ghai, S., I. Ghai \& A.O. Effenberg. 2018. Effect of rhythmic auditory cueing on gait in cerebral palsy: a systematic review and meta-analysis. Neuropsychiatr. Dis. Treat. 14: 43-59.

35. Ghai, S., M.W. Driller \& R.S.W. Masters. 2018. The influence of below-knee compression garments on knee-joint proprioception. Gait. Posture. 60: 258-261.

36. Selfe, J., M. Callaghan, A. McHenry, et al. 2006. An investigation into the effect of number of trials during proprioceptive testing in patients with patellofemoral pain syndrome. J. Orthop. Res. 24: 1218-1224. 37. Zhang, J.-T., A.C. Novak, B. Brouwer, et al. 2013. Concurrent validation of Xsens MVN measurement of lower limb joint angular kinematics. Physiol. Meas. 34: N63.

38. Cooper, G., I. Sheret, L. McMillian, et al. 2009. Inertial sensor-based knee flexion/extension angle estimation. J. Biomech. 42: 2678-2685.

39. Foxe, J.J. 2009. Multisensory Integration: Frequency Tuning of Audio-Tactile Integration. Curr. Biol. 19: R373-R375.

40. Schmitz, G., B. Mohammadi, A. Hammer, et al. 2013. Observation of sonified movements engages a basal ganglia frontocortical network. BMC. Neurosci. 14: 1.

41. Choi, W., G. Lee \& S. Lee. 2015. Effect of the cognitive-motor dual-task using auditory cue on balance of surviviors with chronic stroke: A pilot study. Clin. Rehabil. 29: 763-770.

42. Ghai, S., I. Ghai \& A.O. Effenberg. 2017. Effects of dual tasks and dual-task training on postural stability: a systematic review and meta-analysis. Clin. Interv. Aging. 12: 557-577.

43. Meyer, M., S. Elmer, S. Baumann, et al. 2007. Short-term plasticity in the auditory system: differential neural responses to perception and imagery of speech and music. Restor. Neuro. Neursci. 25: 411-431.

44. Rauschecker, J.P. 2001. Cortical plasticity and music. Ann. N. Y. Acad. Sci. 930: 330-336.

45. Auksztulewicz, R., K.J. Friston \& A.C. Nobre. 2017. Task relevance modulates the behavioural and neural effects of sensory predictions. PLoS. Biol. 15: e2003143.

46. Tremblay, K.L., K. Inoue, K. McClannahan, et al. 2010. Repeated Stimulus Exposure Alters the Way Sound Is Encoded in the Human Brain. PLoS. One. 5: e10283.

47. Wulf, G. \& R.A. Schmidt. 1988. Variability in Practice. J. Mot. Behav. 20: 133-149.

48. Lee, T.D., R.A. Magill \& D.J. Weeks. 1985. Influence of practice schedule on testing schema theory predictions in adults. J. Mot. Behav. 17: 283-299.

49. Lee, T.D. \& R.A. Magill. 1985. Can forgetting facilitate skill acquisition? Differing Perspectives in Motor Learning, Memory, and Control. 3-22.

50. Lage, G.M., H. Ugrinowitsch, T. Apolinario-Souza, et al. 2015. Repetition and variation in motor practice: A review of neural correlates. Neurosci. Biobehav. Rev. 57: 132-141. 
51. Schmuckler, M.A. \& D.T. Jewell. 2007. Infants' visual-proprioceptive intermodal perception with imperfect contingency information. Dev. Psychobiol. 49: 387-398.

52. Schmuckler, M.A. 1995. "Chapter 11 - Self-knowledge of Body Position:: Integration of Perceptual and Action System Information". In Advances in Psychology, Vol. 112. P. Rochat, Ed.: 221-241. NorthHolland.

53. Kirkham, N.Z., J.B. Wagner, K.A. Swan, et al. 2012. Sound support: Intermodal information facilitates infants' perception of an occluded trajectory. Infant. Behav. Dev. 35: 174-178.

54. Henson, R.N., D. Eckstein, F. Waszak, et al. 2014. Stimulus-response bindings in priming. Trends. Cogn. Sci. 18: 376-384.

55. Hecht, H., S. Vogt \& W. Prinz. 2001. Motor learning enhances perceptual judgment: A case for action-perception transfer. Psychol. Res. 65: 3-14.

56. Ghai, S., I. Ghai \& A.O. Effenberg. 2018. "Low road" to rehabilitation: a perspective on subliminal sensory neuroprosthetics. Neuropsychiatr. Dis. Treat. 14: 301.

57. Classen, J., J. Liepert, S.P. Wise, et al. 1998. Rapid plasticity of human cortical movement representation induced by practice. J. Neurophysiol. 79: 1117-1123.

58. Skinner, H.B. \& R.L. Barrack. 1991. Joint position sense in the normal and pathologic knee joint. $J$. Electromyogr. Kinesiol. 1: 180-190.

59. Nagai, T., K.F. Allison, J.L. Schmitz, et al. 2016. "Conscious proprioception assessments in sports medicine: how individuals perform each submodality?". In Sports Medicine: 1-13. SM Online Scientific Resources, Dover, DE.

60. Proske, U. \& S.C. Gandevia. 2012. The proprioceptive senses: their roles in signaling body shape, body position and movement, and muscle force. Physiol. Rev. 92: 1651-1697.

61. Proske, U. 2005. What is the role of muscle receptors in proprioception? Muscle. Nerve. 31: 780787.

62. Proske, U., A. Wise \& J. Gregory. 2000. The role of muscle receptors in the detection of movements. Prog. Neurobiol. 60: 85-96.

63. Munro, B.J., T.E. Campbell, G.G. Wallace, et al. 2008. The intelligent knee sleeve: A wearable biofeedback device. Sens. Actuators. B. Chem. 131: 541-547.

64. Fukuda, T.Y., D. Fingerhut, V.C. Moreira, et al. 2013. Open kinetic chain exercises in a restricted range of motion after anterior cruciate ligament reconstruction: a randomized controlled clinical trial. $\mathrm{Am}$. J. Sports. Med. 41: 788-794.

65. Ghai, S., M. Driller \& I. Ghai. 2017. Effects of joint stabilizers on proprioception and stability: A systematic review and meta-analysis. Phys. Ther. Sport. 25: 65-75.

66. Glass, R., J. Waddell \& B. Hoogenboom. 2010. The Effects of Open versus Closed Kinetic Chain Exercises on Patients with ACL Deficient or Reconstructed Knees: A Systematic Review. N. Am. J. Sports. Phys. Ther. 5: 74-84.

67. Jewiss, D., C. Ostman \& N. Smart. 2017. Open versus Closed Kinetic Chain Exercises following an Anterior Cruciate Ligament Reconstruction: A Systematic Review and Meta-Analysis. J. Sport. Med. 2017. 68. Cho, S., J. Ku, Y.K. Cho, et al. 2014. Development of virtual reality proprioceptive rehabilitation system for stroke patients. Comput. Methods. Programs. Biomed. 113: 258-265. 